\title{
Closure of patent foramen ovale: is the case really closed as well?
}

\section{F A Flachskampf, W G Daniel}

Heart 2005;91:449-450. doi: 10.1136/hrt.2004.052217

$\mathrm{P}$ atent (open) foramen ovale (PFO) is a common anomaly, affecting approximately a quarter of the adult population. In most people PFO never causes a health problem, but paradoxical embolism of venous thrombi with deleterious consequences has been well described in the setting of deep venous thrombosis and right heart pressure overload-that is, after severe pulmonary embolism. ${ }^{1}$ After the initial seminal reports ${ }^{2}$ many studies have confirmed a statistical association between unexplained, presumably embolic ischaemic neurologic events in young patients and the presence of a PFO, indicating that these events might be caused by paradoxical embolism from-mostly clinically inapparent—venous thrombosis.

The difficulty, hence, is to define under which circumstances (other than severe pulmonary embolism) PFO constitutes a risk high enough to warrant treatment, and to define optimal treatment. Unfortunately, this is an exercise in weighing several relatively small risks: the risk of recurrence of an ischaemic event, the risk of anticoagulation treatment, the risk of a procedural complication of closure (for example, device embolisation), and the risk of a post-procedural complication (for example, thromboembolism from a device). Assessment is further complicated by an index event, paradoxical embolism, which is almost never directly observed and usually only inferred. Therefore, neither whether the event is embolic nor whether other embolic sources are "ruled out" constitute iron-clad facts in clinical practice.

\section{OTHER PATHOMECHANISMS}

Other conceivable pathomechanisms have been suggested. In this mini-symposium, Amarenco reports that his group did not find an increased frequency of thrombophilia in patients with presumed paradoxical embolism, nor could they document an increased incidence of Valsalva manoeuvres (p 441). Thus, local formation of thrombus in an atrial septal aneurysm or in the PFO itself is suggested as an alternative pathomechanism to venous thromboembolism. An increased atrial vulnerability (development of atrial fibrillation during electrical stimulation) in patients with PFO or atrial septal aneurysms has also been reported, ${ }^{4}$ which could lead to left atrial thrombus formation due to transient atrial fibrillation. However, a left atrial thrombus in patients with sinus rhythm or a local thrombus within a PFO or within an atrial septal aneurysm (except in the presence of deep venous thrombosis) are exceedingly rare findings in clinical routine, thus making it difficult to follow these hypotheses.

\section{SHOULD PFO BE TREATED?}

Regardless of these uncertainties, from a pragmatic - that is, clinical-point of view, the important question is whether a PFO should be treated and how. This question arises in different scenarios. By far the most important is the detection of a PFO during the cardiologic work-up after a presumably embolic ischaemic neurologic event. Other potential indications involve divers, ${ }^{56}$ in particular professional divers, and patients with a PFO who are at increased risk for venous thrombosis - for example, individuals with a thrombophilia.

Regarding the first scenario (secondary prevention), the following issues should be kept in mind:

- For the cited reasons, PFO as the culprit is often just the least unlikely of a number of possibilities, such extracerebral or intracerebral vascular causes, undetected paroxysmal atrial fibrillation, occult aortic plaque, and others. It seems therefore wise to restrict this diagnosis to patients who are unlikely to have thromboembolic atherosclerotic disease or atrial fibrillation, usually individuals under 60 years of age and without classic risk factors, and have a large PFO, as defined by a large amount of bubbles shunting, colour Doppler, or maximal separation of septum primum and septum secundum after Valsalva, or have an atrial septal aneurysm.

- When considering treatment of an incriminated PFO, the risks of the treatment have to be weighed against the risk of recurrence, lest the cure is worse than the disease. The yearly recurrence rate of neurologic events in young patients after a presumed paradoxical embolism on medical treatment is in the range of $4-12 \% .^{7}$ These rates, however, are heavily influenced by selection and referral bias. In the largest study $(n=267)$ to address this question, the presence of a PFO (with or without concomitant atrial septal aneurysm) conferred a risk of recurrent transient ischaemic attack (TIA) or stroke in the first year of $4.1 \%$ under aspirin. ${ }^{8}$ The treatment options are:

- Anticoagulation, which has been shown in randomised controlled trials to be an effective prophylaxis of deep venous thrombosis and of thromboembolism from atrial fibrillation. Though not rigorously tested, it is believed to be the best management of atheroembolic disease originating in the great vessels. The risks of anticoagulation frequently are overstated. The typically cited risk of major bleeding of approximately $5-8 \%$ per year is far higher than recent data from patients using self administered anticoagulation control indicate-namely a risk of major bleeding in the range of $2 \% .^{9}$ Moreover, emerging options like oral thrombin antagonists may make anticoagulation simpler and safer. The necessary duration of anticoagulation for this indication is unknown and has to be decided individually.

- PFO closure, which entails a finite procedural and postprocedural risk. Ongoing learning curves and device refinement make it difficult to define these risks. A recently published single centre experience over six years reported a procedural complication rate of $6 \% .{ }^{10}$ Even nowadays in experienced hands, it is probably fair to say that complications such as device embolisation and major bleeding are in the range of $1 \% .^{7}$ Moreover, implantation of a closure device also entails a risk of follow up embolic 
Table 1 Suggested criteria for eligibility for interventional PFO closure: 1-4 required

1. Preceding ischaemic neurologic event with probable embolic aetiology 2. Age under 60 years and absence of overt atherosclerotic disease (for example, history of myocardial infarction) or more than one

atherosclerotic risk factor

3. Exclusion of other cardiac (for example, atrial fibrillation, endocarditis, tumour) or vascular (carotid stenosis, spontaneous carotid or vertebral dissection, aortic atheromatosis) embolic sources

4. PFO with more than minimal inducible or spontaneous right-to-left

shunt, in particular in the presence of an atrial septal aneurysm

5. Contraindications against or unwillingness to undergo anticoagulation

events, which in the largest study was $4.3 \%$ in the first year. ${ }^{11}$ Thrombus formation (symptomatic or not) after device closure occurred in $1.7 \%$ during the first six months of follow up in the largest available report, ${ }^{12}$ although most of the more thrombosis-prone devices are not used anymore.

These data, though from different studies and populations, do not support superiority of closure over medical treatment. In the face of such a therapeutic dilemma, we usually turn to randomised trials, and such are indeed underway, as pointed out by Meier in this mini-symposium (p 444), although with substantial recruitment problems due to enthusiasm for closure. However, because both anticoagulation management and closure device technology are "moving targets", it is unlikely that these will provide definitive answers to the points raised, unless they produce uniform and overwhelmingly clear advantages for one strategy. In the meantime, it is prudent to exercise caution in the selection of patients for PFO closure (table 1), and to explain risks and benefits to patients. This is particularly the case when primary prevention of even more nebulous risks is contemplated, such as in divers, in patients with a history of venous thrombosis or a thrombophilia, or for treatment of migraine headache. ${ }^{13}$ In general, we do not think that at this point PFO closure can be recommended for primary prevention, with the possible exception of professional divers.

It is also useful to remember that an important and frequent cause of neurologic events in the young, such as spontaneous carotid and vertebral artery dissection, was virtually unknown 30 years ago, and that very recently mitral valve prolapse has been practically eliminated from the list of high embolic risk lesions. ${ }^{14}$ Though therapeutic closure has become very acceptable because of easier and safer procedures and devices, the case for closure remains open, because the culprit is still not identified clearly enough. For a very small group among the $20-30 \%$ of the population who have a $\mathrm{PFO}$, closure is appropriate-we just don't know exactly for whom.

\section{Authors' affiliations}

F A Flachskampf, W G Daniel, Medizinische Klinik II, Universitätsklinik Erlangen, Germany

Correspondence to: Professor Werner G Daniel, Medizinische Klinik II, Universitätsklinik Erlangen, Ulmenweg18, 91054 Erlangen, Germany; werner.g.daniel@rzmail.uni-erlangen.de

\section{REFERENCES}

1 Konstantinides S, Geibel A, Kasper W, et al. Patent foramen ovale is an important predictor of adverse outcome in patients with major pulmonary embolism. Circulation 1998;97:1946-51.

2 Lechat P, Mas JL, Lascault G, et al. Prevalence of patent foramen ovale in patients with stroke. N Engl J Med 1988;318:1148-52.

3 Webster MWI, Chancellor AM, Smith HJ, et al. Patent foramen ovale in young stroke patients. Lancet 1988;2:11-12.

4 Berthet K, Lavergne T, Cohen A, et al. Significant association of atrial vulnerability with atrial septal abnormalities in young patients with ischemic stroke of unknown cause. Stroke 2000;31:398-403.

5 Knauth M, Ries S, Pohimann S, et al. Cohort study of multiple brain lesions in sport divers: role of a patent foramen ovale. BMJ 1997;314:701-5.

6 Schwerzmann M, Seiler C, Lipp E, et al. Relation between directly detected patent foramen ovale and ischemic brain lesions in sport divers. Ann Intern Med 2001; 134:21-4

7 Khairy P, O'Donnell CP, Landzberg MJ. Transcatheter closure versus medical therapy of patent foramen ovale and presumed paradoxical thromboemboli: a systematic review. Ann Intern Med 2003;139:753-60.

8 Mas JL, Arquizan C, Lamy C, et al for the Patent Foramen Ovale and Atrial Septal Aneurysm Study Group. Recurrent cerebrovascular events associated with patent foramen ovale, atrial septal aneurysm, or both. N Engl J Med 2001;345:1740-6.

9 Ansell J, Hasenkam JM, Jacobson A, et al. Guidelines for implementation of patient self-testing and patient self-management of oral anticoagulation. Int J Cardiol (in press).

10 Windecker S, Wahl A, Nedeltchev K, et al. Comparison of medical treatment with percutaneous closure of patent foramen ovale in patients with cryptogenic stroke. J Am Coll Cardiol 2004;44:750-8.

11 Sievert $\mathrm{H}$, Horvath K Zadan E, et al. Patent foramen ovale closure in patients with transient ischemic attack/stroke. J Interv Cardiol 2001;14:261-6.

12 Krumsdorf U, Ostermayer S, Billinger K, et al. Incidence and clinical course of thrombus formation on atrial septal defect and patent foramen ovale closure devices in 1,000 consecutive patients. J Am Coll Cardiol 2004:43:302-9.

13 Wilmshurst PT, Nightingale S, Walsh KP, et al. Effect on migraine of closure of cardiac right-to-left shunts to prevent recurrence of decompression illness or stroke or for haemodynamic reasons. Lancet 2000;356:1648-51.

14 Gilon D, Buonnano FS, Joffe MM, et al. Lack of evidence of an association between mitral-valve prolapse and stroke in young patients. N Engl J Med 1999;341:8-13. 